\title{
Accuracy and precision of pulse oximeter at different sensor locations in patients with heart failure
}

\author{
Alaa Thabet Hassan, ${ }^{1}$ Soher Mostafa Ahmed, ${ }^{2}$ Azza Salah AbdelHaffeez, ${ }^{3}$ Sherif A.A. Mohamed ${ }^{1}$ \\ ${ }^{1}$ Department of Chest Diseases and Tuberculosis \\ ${ }^{2}$ Department of Internal Medicine \\ ${ }^{3}$ Department of Medical Physiology, Faculty of Medicine, Assiut University, Assiut, Egypt
}

Background: Despite its wide use in clinical practice, few studies have assessed the role of pulse oximetry in patients with heart failure. We aimed to evaluate the accuracy and precision of the pulse oximeter in patients with heart failure and to determine this accuracy at three different sensor locations.

Methods: Comparison of pulse oximetry reading $\left(\mathrm{SpO}_{2}\right)$ with arterial oxygen saturation $\left(\mathrm{SaO}_{2}\right)$ was reported in 3 groups of patients with heart failure (HF); those with ejection fraction (EF) $>40 \%$, those with EF $<40 \%$, and those with acute HF (AHF) with ST and non-ST segment elevation acute myocardial infarction (STEMI and non-STEMI).

Results: A total of 235 patients and 90 control subjects were enrolled. There were significant differences in $\mathrm{O}_{2}$ saturation between control and patients' groups when $\mathrm{O}_{2}$ saturation is measured at the finger and toe, but not the ear probes; $\mathrm{p}=0.029, \mathrm{p}=0.049$, and 0.051 , respectively. In $\mathrm{HF}$ with $\mathrm{EF}>40 \%$ and $\mathrm{AHF}$ with $\mathrm{O}_{2}$ saturations $>90 \%$, finger oximetry is the most accurate and reliable, while in $\mathrm{HF}$ with $\mathrm{EF}<40 \%$ and in patients with $\mathrm{AHF}$ with $\mathrm{O}_{2}$ saturations $<90 \%$, ear oximetry is the most accurate.

Conclusion: Pulse oximetry is a reliable tool in assessing oxygen saturation in patients with heart failure of different severity. In $\mathrm{HF}$ with $\mathrm{EF}>40 \%$ and in $\mathrm{AHF}$ with $\mathrm{O}_{2}$ saturations $>90 \%$, finger oximetry is the most accurate and reliable, while in $\mathrm{HF}$ with $\mathrm{EF}<40 \%$ and in patients with $\mathrm{AHF}$ with $\mathrm{O}_{2}$ saturations < $90 \%$, ear oximetry is the most accurate. Further studies are warranted.

Key words: Pulse oximeter; heart failure; accuracy; sensor; location; myocardial infarction; evaluation.

Correspondence: Dr. Sherif A.A. Mohamed, Associate Professor of Pulmonary Medicine. Department of Chest Diseases and Tuberculosis, Faculty of Medicine, Assiut University, 71516 Assiut, Egypt.

Tel. +20.88.241371, +20.966.538618846 - Fax: +20.88.241333327. E-mail: saawm220@gmail.com

Contributions: ATH, SMA, SAAM, contributed to the draft of the submitted article, revised it critically for important content, and made substantial contributions to conception and design, as well as data acquisition, analysis, and interpretation; SAAM, ASA, contributed to the draft of the submitted article and made substantial contributions to acquisition, analysis, and interpretation of clinical and physiological data. All authors have provided approval of the manuscript to be published and have agreed to be accountable for all aspects of the work in ensuring that questions related to the accuracy or integrity of any part of the work are appropriately investigated and resolved.

Conflict of interest: The authors declare that they have no competing interests, and all authors confirm accuracy.

Funding: This research did not receive any specific grant from funding agencies in the public, commercial, or not-for-profit sectors.

Availability of data and materials: The datasets used and/or analyzed during the current study are available from the corresponding author on reasonable request.

Ethics approval and consent to participate: The study was approved by the Ethical Committee of the Faculty of Medicine, Assiut University (approval number: 5441).

Consent for publication: Not applicable. 


\section{Introduction}

When arterial oxyhemoglobin saturation is measured noninvasively by pulse oximetry, it is referred to as $\mathrm{SpO}_{2}$, and it is a measure of the amount of oxygen-carrying hemoglobin in the blood relative to the amount of hemoglobin not carrying oxygen [1]. The gold standard for determining $\mathrm{SpO}_{2}$ is the arterial blood gas analysis (ABGA) or rather CO-oximetry with direct measurement of functional oxygen saturation [2]. Despite that this technique is more accurate, still it is invasive, intermittent, and cost-intensive. Pulse oximetry determines the functional oxygen saturation noninvasively, continuously, and in real-time, and it is much cheaper $[1,2]$. Pulse oximetry has become a standard monitoring technique during perioperative anesthesia, intensive care therapy, and emergency medical treatment of severely ill or injured patients [3].

Multiple sensor sites have been evaluated and analyzed concerning the accuracy and precision of pulse oximetry [1]. It was observed that, in critically ill and mechanically ventilated patients, fingers and toes are the recommended sensor sites for pulse oximetry measurement for routine clinical use. If the measurement is performed at the ear lobe, there was a tendency for lower accuracy and precision, but the difference is usually not clinically significant $[1,3]$.

Heart failure (HF) has been traditionally described as acute or chronic, but this can be confusing and should be used to describe time, rather than severity. Acute heart failure (AHF) can present as new-onset heart failure in people without known cardiac dysfunction, or as acute decompensation of chronic heart failure [4]. Few studies have assessed the role of pulse oximetry in acute heart failure [5,6]. Masip and coworkers [5] had concluded that baseline oxygen saturation by pulse oximetry is useful in establishing the diagnosis and severity of heart failure in acute settings such as myocardial infarction and may have prognostic implications. Also, they observed that the diagnosis of AHF may be suspected when baseline oxygen saturation by pulse oximetry is $<93 \%$ [5].

Therefore, the aims of the current study were to evaluate the accuracy and precision of the pulse oximeter in patients with heart failure including those with acute HF, and to determine and compare this accuracy at three different sensor locations.

\section{Materials and Methods}

The current study was conducted at Assiut University Hospital. Enrolment criteria included all patients diagnosed with heart failure and admitted to the Intermediate Cardiology Care Unit (ICCU), Internal Medicine Department, from July $1^{\text {st }}, 2018$ to $31^{\text {st }}$ December 2019. Exclusion criteria included: i) patients with core body temperature $<36^{\circ} \mathrm{C}$; ii) patients with metabolic acidosis (serum lactate level $>2 \mathrm{mmol} / \mathrm{L}$ ); iii) those who developed cardiogenic shock; and iv) patients with diseases or conditions known to affect pulse oximeter accuracy, e.g., sickle cell disease, methemoglobinemia, carboxyhemoglobinemia. Enrolled subjects were subjected to full clinical evaluation including smoking history and medical comorbidities. Age and gender-matched control groups were enrolled.

The pulse oximeter used was a Philips M1020A pulse oximetry module (Philips Medical Systems, Eindhoven, Netherlands). An appropriate-sized pulse oximeter sensor (Phillips M1020A/ M1192A/ M1194A/M1195A) was applied to the finger, on the toe, and the ear lobe.

Patients were continuously monitored by pulse oximetry during their stay in the ICCU. The target $\mathrm{SpO}_{2}$ was 'morning baseline $\mathrm{SpO}_{2}$ ', which was registered by nurses in awake patients on the morning round, usually after chest radiography, and taking the most stable value on room air. For patients receiving oxygen therapy, it was recorded when a steady value was reached, after several minutes of oxygen discontinuation.

At the same time, an arterial blood sample was drawn. A single reading of $\mathrm{SpO}_{2}$ was taken in individual sensor locations after the pulse oximeters had achieved optimal plethysmography signals and heart rates matching with the electrocardiogram (ECG) monitor (CMS; Philips Medical Systems). In those patients with variable heart rate(s), readings of $\mathrm{SPO}_{2}$ were taken after control of their abnormal heart rates. Simultaneously, an arterial blood gas (ABG) analysis was done for co-oximetric measurement of $\mathrm{SaO}_{2}$ using an ABL 800 FLEX ABG machine (Radiometer America Inc., Cleveland, OH, USA). The core temperature, arterial pressure, heart rate, and $\mathrm{ABG}$ value of hemoglobin were recorded simultaneously.

Comparison of pulse oximetry reading $\left(\mathrm{SpO}_{2}\right)$ with arterial oxygen saturation $\left(\mathrm{SaO}_{2}\right)$ was reported in terms of bias and precision as described by Bland and Altman [7]. Bias is the difference between the specific body location $\mathrm{SpO}_{2}$ (finger, toe, ear) and $\mathrm{SaO}_{2}$ (i.e., $\mathrm{SpO}_{2}-\mathrm{SaO}_{2}$ ) and precision is the \pm 1 standard deviation of the difference [7]. A low bias in a sensor site implies that the pulse oximeter sensor gives a more accurate reading at that site and vice versa. Precision implies the reproducibility of the measurement.

\section{Echocardiography}

Echocardiographic examination was carried out using commercially available cardiac ultrasound machines (4500 echocardiography machine with a $3.5 \mathrm{MHz}$ electronic probe; HewlettPackard, Palo Alto, CA, USA). All patients and control subjects underwent a two-dimensional, pulse-waved, color-flow Doppler echocardiography (ATL 9) of the right side of the heart (right atrium, tricuspid valve, right ventricle, pulmonary valve, and artery) in parasternal long-axis, short-axis, short-axis cross-section of the base of the heart, and apical four-chamber view, to evaluate the right heart performance and to calculate the mean PPA. When tricuspid regurgitation was recorded with color-flow Doppler, the maximum velocity $(\mathrm{V})$ of tricuspid incompetence was calculated with a continuous Doppler study of at least four consecutive beats. Right ventricle pressure (RVP) was derived using the equation $\mathrm{RVP}=4 \mathrm{~V} 2+\mathrm{RAP}(\mathrm{V}=$ the maximum velocity of tricuspid incompetence, $\mathrm{RAP}=$ right atrial pressure). The estimated RVP is considered to represent the PPA if there is no evidence of pulmonary valvular dysfunction.

The patients were divided into three groups: i) those with ejection fraction (EF) more than $40 \%$ (group A); ii) those with EF less than $40 \%$ (group B); and iii) those with acute HF with ST and nonST segment elevation acute myocardial infarction (STEMI and non-STEMI) (group C). The study was approved by the Ethical Committee of the Faculty of Medicine (Approval number 5441), Assiut University, and written informed consent was provided by all patients.

\section{Statistical analysis}

Data were described as mean \pm standard deviation (SD) and frequencies if they are quantitative or qualitative, respectively. Mann-Whitney test and Wilcoxon Signed Ranks test were used for comparison of quantitative variables among two independent and dependent groups, respectively. Chi-Square test was used for comparison of the distribution of qualitative variables among different groups. Correlation between continuous variables was performed using Spearman's correlation coefficient. Kruskal-Wallis test was used to compare the bias of two sensor sites statistically. A $p<0.05$ was interpreted as significant. The SPSS (Statistical Package for Social Science) software v. 20 was used for statistical analysis. 


\section{Results}

\section{Demographic characteristics}

A total of 235 patients and 90 control subjects were enrolled. Patients included 130 (55.3\%) females and 105 (44.7\%) males. Patients included 85, 81, and 69 subjects in groups A, B, and C, respectively. Among those in group $\mathrm{C}$, the mean $\mathrm{EF}$ was $38.2 \pm 11.5$, and 12/69 (17\%) patients had systemic hypotension. Comparison between the patients' and control groups revealed no significant differences. Table 1 shows these demographic data and associated comorbidities.

\section{Oxygen saturation measured by pulse oximetry and ABGs in different locations}

There was no significant difference in $\mathrm{O}_{2}$ saturation between the control and the 3 patients' groups $(\mathrm{p}=0.056)$. With regards to locations, there were significant differences in $\mathrm{O}_{2}$ saturation between control and patients' groups when $\mathrm{O}_{2}$ saturation was measured at the finger and toe, but not the ear probes; $p=0.029$, $\mathrm{p}=0.049$, and 0.051 , respectively. These results are shown in Table 2 and Figure 1. It is of note that Table 2 showed that values of oxygen saturation measured by pulse oximetry in the ear are homogeneous in all 4 groups (controls, A, B, and C), while those values measured in finger or toe are not homogenous. This could explain overestimated desaturation in groups $\mathrm{B}$ and $\mathrm{C}$, in the sensor locations of the finger and toe.

\section{Mean bias in different oxygen saturations in different locations}

The mean bias was measured for different oxygen saturations in different locations and among the patients' groups (Table 3; Figures 2-4).

In group $\mathrm{A}$, the finger sensor was found to have the least bias, hence the most accuracy. The value of mean $\mathrm{SaO}_{2}$ in the ear sensor was higher than the $\mathrm{SaO}_{2}$ in the $\mathrm{ABG}$ (positive). Thus, it can be said that the pulse oximeter in the ear overestimates $\mathrm{SaO}_{2}$ in group A patients. For the toe sensor, the pulse oximeter also overestimates $\mathrm{SaO}_{2}$ in this group of patients (Table 3 and Figure 2). In Group B, the ear sensor had the least bias, hence the most accuracy with different $\mathrm{O}_{2}$ saturations; while the pulse oximeter underestimates the $\mathrm{SaO}_{2}$ in finger and toe sensors especially in $\mathrm{O}_{2}$ saturations $<90 \%$ (Table 3, Figure 3). In Group C, the ear sensor had the least bias, hence the most accuracy with $\mathrm{O}_{2}$ saturations $<90 \%$, the finger sensor was found to have the least bias and hence the most accuracy with $\mathrm{O}_{2}$ saturations $>90 \%$. For the toe sensor, the pulse oximeter underestimates $\mathrm{SaO}_{2}$. Furthermore, it can be said that

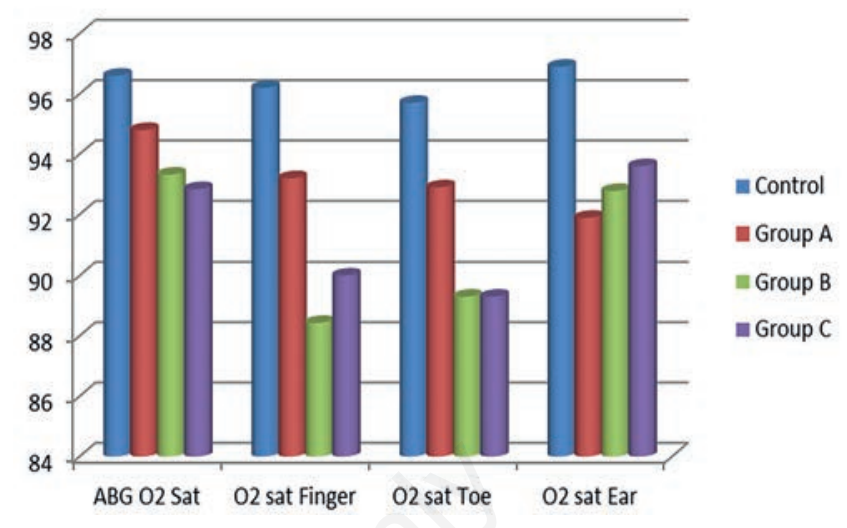

Figure 1. Mean $\mathrm{O}_{2}$ saturation measured by pulse oximetry and arterial blood gases $(\mathrm{ABG})$ in different locations.

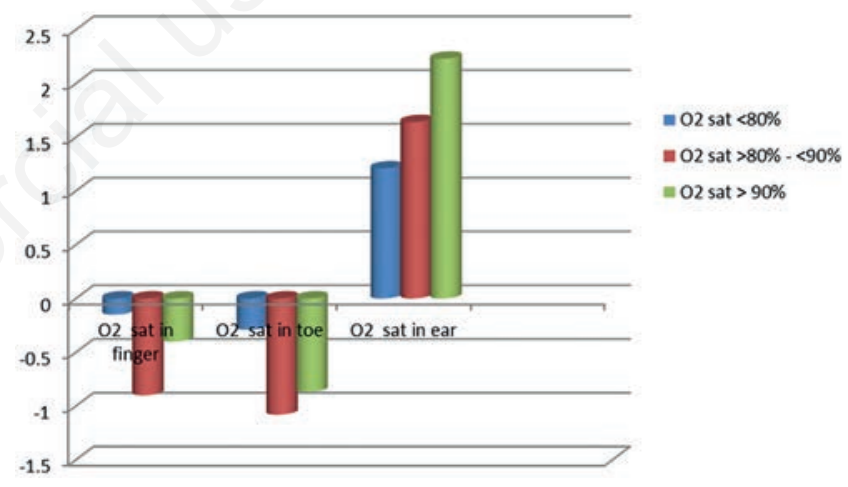

Figure 2. Pattern of mean bias in oxygen saturation measured with pulse oximeter in Group A.

Table 1. Demographic data of the study and control groups.

\begin{tabular}{|c|c|c|c|}
\hline & $\begin{array}{c}\text { Study group (n=235) } \\
\text { N (\%) }\end{array}$ & $\begin{array}{c}\text { Control group }(\mathrm{n}=90) \\
\text { N (\%) }\end{array}$ & $\mathbf{p}$ \\
\hline $\begin{array}{l}\text { Age (years) } \\
\quad \text { Mean } \pm \text { SD } \\
\text { Median (range) }\end{array}$ & $\begin{array}{l}56.01 \pm 12.38 \\
58(22-76)\end{array}$ & $\begin{array}{l}53.25 \pm 12.16 \\
55(26-76)\end{array}$ & 0.815 \\
\hline $\begin{array}{l}\text { Gender } \\
\quad \text { Female } \\
\text { Male }\end{array}$ & $\begin{array}{l}130(55.3) \\
105(44.7)\end{array}$ & $\begin{array}{l}48(53.3) \\
42(46.7)\end{array}$ & 0.804 \\
\hline $\begin{array}{l}\text { Comorbidities } \\
\text { DM } \\
\text { HTN } \\
\text { Hypothyroidism } \\
\text { Hyperlipidemia } \\
\text { Hyperthyroidism } \\
\text { Bronchial asthma }\end{array}$ & $\begin{array}{c}70(29.8) \\
91(38.7) \\
12(5.1) \\
126(53.6) \\
8(3.4) \\
45(19.1)\end{array}$ & $\begin{array}{c}30(33.3) \\
28(31.1) \\
4(4.4) \\
35(38.9) \\
3(3.3) \\
15(16.7)\end{array}$ & $\begin{array}{l}0.591 \\
0.247 \\
1.000 \\
0.019 \\
1.000 \\
0.749\end{array}$ \\
\hline
\end{tabular}

SD, standard deviation; DM, diabetes mellitus; HTN, hypertension. 
findings in Table 3 confirm that, the obtained measures from the finger and toe sensor underestimate the $\mathrm{SaO}_{2}$ with a direct correlation to the severity of $\mathrm{SaO}_{2}$ (the greater the severity is the greater the overestimate); on the contrary, the ear sensor overestimates the $\mathrm{SaO}_{2}$ with an inverse correlation in respect to the severity (the more normal the subject is the greater the overestimate).

Thus, it can be concluded that in patients with AHF, pulse oximeter in the finger underestimates $\mathrm{SaO}_{2}$ at saturations less than $90 \%$, while in those saturations more than $90 \%$ finger oximetry readings were almost the same as the $\mathrm{SaO}_{2}$ in $\mathrm{ABG}$. Thus, finger oximetry is the most accurate with $\mathrm{O}_{2}$ saturations of more than $90 \%$ in patients with AHF. On the other hand, in patients with AHF ear pulse oximeter mildly overestimates $\mathrm{SaO}_{2}$, and the toe oximeter underestimates $\mathrm{SaO}_{2}$ (Table 3, Figure 4).

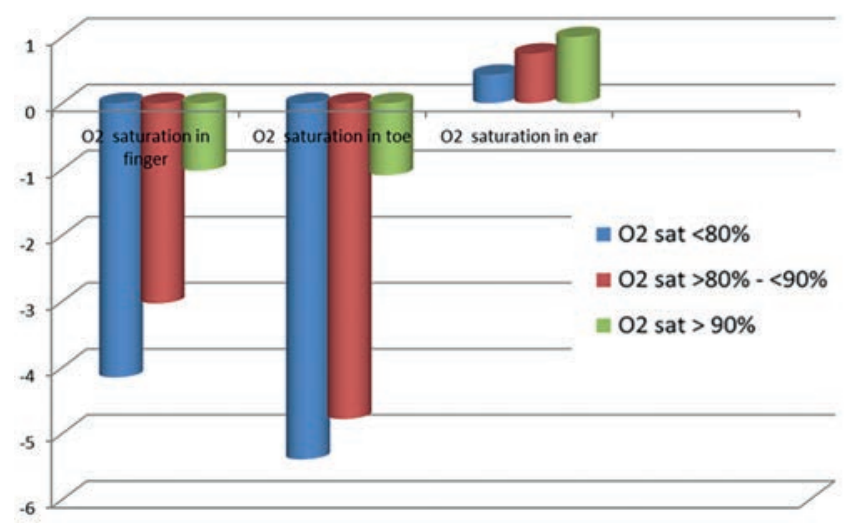

Figure 3. Pattern of mean bias in oxygen saturation measured with pulse oximeter in Group B.

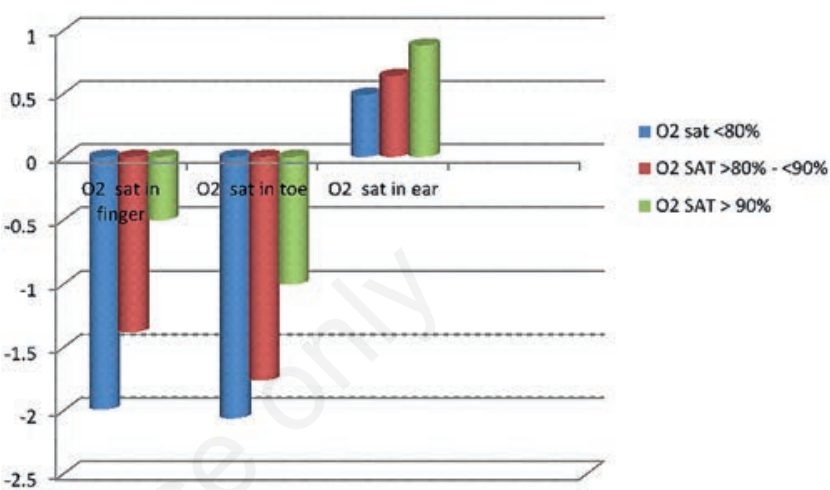

Figure 4. Pattern of mean bias in oxygen saturation measured with pulse oximeter in Group C.

Table 2. Oxygen saturation measured by pulse oximetry and ABGs and in different locations among different groups of HF patients.

\begin{tabular}{lccccc} 
& Control & $\begin{array}{c}\text { Group A } \\
(\mathbf{E F}>\mathbf{4 0 \% )}\end{array}$ & $\begin{array}{c}\text { Group B } \\
(\mathbf{E F}<40 \%)\end{array}$ & $\begin{array}{c}\text { Group C } \\
\text { (Acute HF) }\end{array}$ \\
$\mathrm{O}_{2}$ sat in $\mathrm{ABGs}$ & $96.6 \pm 3.14$ & $94.8 \pm 5.1$ & $93.32 \pm 3.63$ & $92.84 \pm 7.45$ & p \\
$\mathrm{O}_{2}$ sat in finger & $96.2 \pm 4.2$ & $93.2 \pm 5.4$ & $88.43 \pm 8.2$ & $90 \pm 6.3$ & 0.056 \\
\hline $\mathrm{O}_{2}$ sat in toe & $95.7 \pm 4,7$ & $92.9 \pm 2.8$ & $89.3 \pm 6.1$ & $89.3 \pm 10.6$ & $0.029^{*}$ \\
$\mathrm{O}_{2}$ sat in ear & $96.9 \pm 2.8$ & $91.89 \pm 3.5$ & $92.78 \pm 8.7$ & $93.6 \pm 6.2$ & $0.049 *$ \\
$\mathrm{p}$ & 0.09 & 0.054 & $0.005^{*}$ & $0.008^{*}$ \\
\hline
\end{tabular}

ABGs, arterial blood gases; $\mathrm{HF}$, heart failure; $\mathrm{EF}$, ejection fraction; $\mathrm{O}_{2}$ sat, oxygen saturation.

Table 3. Mean bias in different oxygen saturations measured by pulse oximetry in comparison to arterial blood gases among different groups of HF patients.

\begin{tabular}{|c|c|c|c|c|c|}
\hline & & $\begin{array}{c}\text { Group A } \\
\text { Mean bias } \pm \text { SD }\end{array}$ & $\begin{array}{c}\text { Group B } \\
\text { Mean bias } \pm \text { SD }\end{array}$ & $\begin{array}{c}\text { Group C } \\
\text { Mean bias } \pm \text { SD }\end{array}$ & $\mathbf{p}$ \\
\hline $\mathrm{O}_{2}$ saturation in the finger & $\begin{array}{c}\mathrm{O}_{2} \text { sat }<80 \% \\
\mathrm{O}_{2} \text { sat }>80 \%-<90 \% \\
\mathrm{O}_{2} \text { sat }>90 \%\end{array}$ & $\begin{array}{l}-0.15 \pm 1.26 \\
-0.9 \pm 1.01 \\
-0.4 \pm 0.43\end{array}$ & $\begin{array}{l}-4.15 \pm 3.15 \\
-3.03 \pm 2.49 \\
-1.02 \pm 1.28\end{array}$ & $\begin{array}{c}-1.99 \pm 1.75 \\
-1.38 \pm 1.01 \\
-0.5 \pm 0.28\end{array}$ & $\begin{array}{l}0.027^{*} \\
0.041^{*} \\
0.009 *\end{array}$ \\
\hline $\mathrm{O}_{2}$ saturation in the toe & $\begin{array}{c}\mathrm{O}_{2} \text { sat }<80 \% \\
\mathrm{O}_{2} \text { sat }>80 \%-<90 \% \\
\mathrm{O}_{2} \text { sat }>90 \%\end{array}$ & $\begin{array}{l}-0.29 \pm 0.22 \\
-1.08 \pm 0.05 \\
-0.87 \pm 0.77\end{array}$ & $\begin{array}{l}-5.39 \pm 4.25 \\
-4.78 \pm 3.96 \\
-1.09 \pm 0.65\end{array}$ & $\begin{array}{c}-2.06 \pm 1.83 \\
-1.76 \pm 1.30 \\
-1 \pm 0.02\end{array}$ & $\begin{array}{c}0.03^{*} \\
0.040^{*} \\
0.09\end{array}$ \\
\hline $\mathrm{O}_{2}$ saturation in the ear & $\begin{array}{c}\mathrm{O}_{2} \text { sat }<80 \% \\
\mathrm{O}_{2} \text { sat }>80 \%-<90 \% \\
\mathrm{O}_{2} \text { sat }>90 \%\end{array}$ & $\begin{array}{l}1.21 \pm 0.89 \\
1.64 \pm 1.23 \\
2.23 \pm 0.76\end{array}$ & $\begin{array}{c}0.43 \pm 0.35 \\
0.75 \pm 0.54 \\
1.0 \pm 0.91\end{array}$ & $\begin{array}{l}0.49 \pm 0.23 \\
0.64 \pm 0.45 \\
0.88 \pm 0.62\end{array}$ & $\begin{array}{c}0.06 \\
0.075 \\
0.049 *\end{array}$ \\
\hline p & & $0.009^{*}$ & $0.000 *$ & $0.01^{*}$ & \\
\hline
\end{tabular}

SD, standard deviation; 02 sat, oxygen saturation; ${ }^{*} \mathrm{p}<0.05$. 


\section{Discussion}

In the current study, we have observed that pulse oximetry is a reliable tool in assessing oxygen saturation in patients with heart failure of different severity. In heart failure with $\mathrm{EF}>40 \%$ and $\mathrm{AHF}$ with $\mathrm{O}_{2}$ saturations more than $90 \%$, finger oximetry is the most accurate and reliable, while in $\mathrm{HF}$ with $\mathrm{EF}<40 \%$ and in patients with AHF with $\mathrm{O}_{2}$ saturations less than $90 \%$, ear oximetry is the most accurate.

Considering the limitations and advantages of pulse oximetry in various parts of the body, the importance of accurate detection of hypoxemia, and the scarcity of studies about the ideal method of pulse oximetry in patients admitted to intermediate or intensive care units for heart failure [5,6], the present study was conducted to determine the accuracy of pulse oximetry probes of the finger, toe, and earlobe in the detection of oxygen saturation in the patients with heart failure of different grades. We hypothesized that different locations of the pulse oximeter among different grades of heart failure could affect assessment and hence management of those patients. Pulse oximeters measure the difference in light absorption of oxygenated and reduced hemoglobin and then calculate the percentage of hemoglobin that is saturated with oxygen $[1,8,9]$.

It is thought that sensor location may affect the accuracy and precision of pulse oximetry, so multiple sensor sites have been evaluated and analyzed [1-3]. It was observed that, in critically ill and mechanically ventilated patients, fingers and toes are the recommended sensor sites for pulse oximetry measurement for routine clinical use $[1,3]$.

Patients with heart failure, especially those with acute HF due to AMI, represent a particular group of patients where urgent and meticulous monitoring are required, hence rapid yet accurate management decisions could be taken. In these situations, pulse oximetry has an important implication. In general, HF patients who do not have comorbid lung disease do not manifest hypoxemia except in severe acute decompensation. Patients with severe HF may have signs and symptoms ranging from severe hypoxemia, or even hypoxia, along with hypercapnia, to decreased vital capacity and poor ventilation [10]. Although arterial blood gas (ABG) measurement is more accurate than pulse oximetry for measuring oxygen saturation, it is unclear if $\mathrm{ABG}$ results add any clinical utility to pulse oximetry. In the setting of acute heart failure, ABG measurement is rarely performed. Indications include severe respiratory distress, documented hypoxemia by pulse oximetry not responsive to supplemental oxygen, and evidence of acidosis by serum chemistry findings or elevated lactate levels $[4,5,10]$.

Pulse oximetry is highly accurate at assessing the presence of hypoxemia and, therefore, the severity of acute heart failure presentations. Patients with mild to moderate acute heart failure may show modest reductions in oxygen saturation, whereas patients with severe heart failure may have severe oxygen desaturation, even at rest. Pulse oximetry is also useful for monitoring the patient's response to supplemental oxygen and other therapies [4$6,10]$.

In a prospective observational cohort study of 220 consecutive patients with acute myocardial infarction, Masip and co-workers [5] observed that the utility of baseline $\mathrm{SpO}_{2}$ as a complementary tool to establish the diagnosis and severity of AHF. The advantages of pulse oximetry in this setting are, being non-invasive, it can be monitored continuously, and is not affected by interobserver or intraobserver variability. The authors considered that finding of a baseline $\mathrm{SpO}_{2}$ lower than 93 may be considered a signal of AHF and a warning to clinicians about this complication which may be especially useful between rounds [5].
Various studies have reported a decline in accuracy and precision of pulse oximetry when the $\mathrm{SaO}_{2}$ decreases below various cutoff values $(<90 \%$ or $<80 \%)$ [11,12]. Different sensors also affect the accuracy of $\mathrm{SpO}_{2}$ measurements. Clayton and colleagues found that the overall rankings were much better for the finger sensors in patients with poor peripheral perfusion [13]. Our results showed that there were significant differences in $\mathrm{O}_{2}$ saturation between control and patients' groups when $\mathrm{O}_{2}$ saturation is measured at the finger and toe, but not the ear probes. Moreover, we have observed that in heart failure with $\mathrm{EF}>40 \%$ and AHF with $\mathrm{O}_{2}$ saturations more than $90 \%$, finger oximetry is the most accurate and reliable, while in $\mathrm{HF}$ with $\mathrm{EF}<40 \%$ and in a patient with AHF with $\mathrm{O}_{2}$ saturations less than $90 \%$, ear oximetry is the most accurate. Ambient light, skin pigmentation, dyshemoglobinaemia, low peripheral perfusion states, and motion artifact can affect the performance of pulse oximeters $[14,15]$. At low levels of saturation $\left(\mathrm{SaO}_{2}\right.$ below $80 \%$ ), pulse oximetry is not as accurate as at higher saturations [12]. Although the exact mechanism is not known, various investigators have found that $\mathrm{SpO}_{2}$ overestimates $\mathrm{SaO}_{2}$ in polycythemia and underestimates $\mathrm{SaO}_{2}$ with anemia [16,17].

While studying the effect of sensor location on pulse oximeter accuracy and precision in cyanotic children, Sedaghat-Yazdi et al. [18] found that there are no significant differences in bias and precision between finger and toe sensors regardless of $\mathrm{SaO}_{2}$ values. They also found that sensor locations with the worst accuracy and precision were the sole and palm when $\mathrm{SaO}_{2}$ was $<90 \%$. On the other hand, it was observed that sole was the most accurate site of sensor location in cyanotic heart disease pediatric patients, in a study by Das and co-workers [19]. The authors could also re-establish the finding that at low saturation states, pulse oximetry accuracy deteriorates and tends to overestimate the $\mathrm{SaO}_{2}$. Moreover, in their study, in terms of reproducibility, the best sensor site could not be determined definitely and consistently [19]. Furthermore, results of the current study highlight those obtained from other reports $[3,11,12,18,19]$, that toe probes offer no advantages over other locations among adults, compared to pediatrics.

It should be emphasized that the pulse oximeter, like any clinical monitoring tool, must be used correctly and the results interpreted properly. Despite the fact that the pulse oximeter has the advantages of being a non-invasive, easy-to-use tool that provides useful and continuous information about the oxygenation of a patient's blood, clinicians must recognize the limitations of pulse oximetry. The pulse oximeter is an additional assessment tool, not a substitute for other elements of the assessment. Pulse oximeters can increase patient safety by alerting the clinician to hypoxia. However, the reading should always be interpreted in association with the patient's clinical condition, particularly in critically ill patients like those with acute HF. Limitations of the current study include relatively low numbers of enrolled patients. Further studies with larger numbers are needed.

\section{Conclusions}

Pulse oximetry is a reliable tool in assessing oxygen saturation in patients with heart failure of different severity. An understanding of the bias and precision of pulse oximetry at various sensor locations would be effective in the management of patients with heart failure. In heart failure with $\mathrm{EF}>40 \%$ and $\mathrm{AHF}$ with $\mathrm{O}_{2}$ saturations of more than $90 \%$, finger oximetry is the most accurate and reliable. In $\mathrm{HF}$ with $\mathrm{EF}<40 \%$ and in a patient with $\mathrm{AHF}$ with $\mathrm{O}_{2}$ saturations less than $90 \%$, ear oximetry is the most accurate. Further studies are warranted. 


\section{Acknowledgments}

The authors thank Mr Omar Sherif Mohamed (omard114@gmail.com), the research coordinator, Department of Chest Diseases and Tuberculosis, Faculty of Medicine, Assiut University, for his great help in this manuscript.

\section{References}

1. DeMeulenaere S. Pulse Oximetry: Uses and Limitations. JNP 2007;3:312-7.

2. Hinkelbein J, Genzwuerker H, Fiedler F. Reliability and variability of oxygen saturation determined by pulse oximetry in intensive care patients. Anesthesiology 2003;99:A452.

3. Hinkelbein J, Hose D, Fiedler F. Comparison of three different sensor sites for pulse oximetry in critically ill patients. Int $\mathrm{J}$ Intensive Care 2005;12:159-63.

4. National Clinical Guideline Centre (UK). Acute heart failure: Diagnosing and managing acute heart failure in adults. London: National Institute for Health and Care Excellence; 2014.

5. Masip J, Gayà M, Páez J, Betbesé A, Vecilla F, Manresa R, et al. Pulse oximetry in the diagnosis of acute heart failure. Rev Esp Cardiol (Engl Ed) 2012;65:879-84.

6. Sepehrvand N, Alemayehu W, Rowe BH, McAlister FA, van Diepen S, Stickland M, et al. High vs. low oxygen therapy in patients with acute heart failure: HiLo-HF pilot trial. ESC Heart Fail 2019;6:667-77.

7. Bland JM, Altman DG. Statistical methods for assessing agreement between two methods of clinical measurement. Lancet 1986;1:307-10.

8. Brand T, Brand M, Jay G. Enamel nail polish does not interfere with pulse oximetry among normoxic volunteers. J Clin Monit Comput 2002;17:93-6.
9. Goldman JM, Petterson MT, Kopotic RJ, Barker SJ. Masimo signal extraction pulse oximetry. J Clin Monit Comput 2000; $16: 475-83$.

10. Dumitru L, Baker MM. Heart failure. Medscape [Internet]. Available from https://emedicine.medscape.com/article/ 163062-workup\#c11

11. Torres A Jr, Skender KM, Wohrley JD, Aldag CJ, Raff GW, Bysani GK, et al. Pulse oximetry in children with congenital heart disease: Effects of cardiopulmonary bypass and cyanosis. J Intensive Care Med 2004;19:229-34.

12. Schmitt HJ, Schuetz WH, Proeschel PA, Jaklin C. Accuracy of pulse oximetry in children with cyanotic congenital heart disease. J Cardiothorac Vasc Anesth 1993;7:61-5.

13. Clayton D, Webb RK, Ralston AC, Duthie D, Runciman WB. Pulse oximeter sensors: a comparison between finger, nose, ear, and forehead sensors under conditions of poor perfusion. Anaesthesia 1991;46:260-5.

14. Trivedi NS, Ghouri AF, Shah NK, Lai E, Barker SJ. Effects of motion, ambient light, and hypoperfusion on pulse oximeter function. J Clin Anesth 1997;9:179-83.

15. Zeballos RJ, Weisman IM. Reliability of noninvasive oximetry in black subjects during exercise and hypoxia. Am Rev Respir Dis 1991;144:1240-4.

16. Volgyesi GA, Kolesar R, Lerman J. An in vitro model for evaluating the accuracy of pulse oximeters. Can J Anaesth 1990;37:S67.

17. Kolesar R, Volgyesi G, Lerman J. Effect of haemoglobin concentration on the accuracy of pulse oximetry. Can J Anaesth 1990;37:S88.

18. Sedaghat-Yazdi F, Torres A Jr, Fortuna R, Geiss DM. Pulse oximeter accuracy and precision affected by sensor location in cyanotic children. Pediatr Crit Care Med 2008;9:393-7.

19. Das J, Aggarwal A, Aggarwal NK. Pulse oximeter accuracy and precision at five different sensor locations in infants and children with cyanotic heart disease. Indian J Anaesth 2010;54:531-4. 